\title{
Nutcracker syndrome associated with celiacomesentric trunk anomaly: case report
}

This article was published in the following Dove Press journal: International Journal of Nephrology and Renovascular Disease

\author{
Nabil A Al-Zoubi' \\ Ibrahim F Al-Ghalayini ${ }^{1,2}$ \\ Radwan Al-Okour ${ }^{1,2}$ \\ 'Department of Surgery, ${ }^{2}$ Division of \\ Urology, Jordan University of Science \\ and Technology, Irbid, Jordan
}

Introduction: Nutcracker syndrome is a rare disease entity that is caused by entrapment of the left renal vein between the aorta and superior mesenteric artery, usually due to abnormal branching of the superior mesenteric artery from the aorta causing renal venous hypertension. The symptoms vary from asymptomatic hematuria to severe pelvic congestion. Celiacomesenteric trunk anomaly is a rare variation of splanchnic artery anomaly that occurs when the celiac trunk and superior mesenteric arteries have a common origin from the aorta. A disease involving the rarely encountered celiacomesenteric trunk anomaly is extremely uncommon. To our knowledge, association between nutcracker syndrome and celiacomesentric trunk anomaly has not been reported in the literature.

Case presentation: A 14-year-old boy with no significant past medical history presented with a 3-year painless hematuria. CT-angiogram revealed anterior nutcracker syndrome with celiacomesenteric trunk anomaly. The patient was managed conservatively with close follow-up. Conclusion: Nutcracker syndrome associated with celiacomesenteric trunk anomaly is extremely uncommon and is a rare cause of hematuria in children. Whether this abnormal anatomy is the cause of nutcracker syndrome or just an association should be investigated. Moreover, awareness of this anatomical variation may help in planning therapeutic options and reducing the chance of surgical iatrogenic injuries.

Keywords: nutcracker syndrome, celiacomesenteric trunk, hematuria in children

\section{Introduction}

Nutcracker phenomenon (NCP) is a rare vascular variation that is caused by contraction of the left renal vein (LRV), usually between the superior mesenteric artery (SMA) and the aorta (anterior nutcracker) or in a rare variant between the abdominal aorta and the vertebral spine (posterior nutcracker). ${ }^{1,2}$ When clinically manifested as proteinuria, hematuria, flank pain, pelvic congestion in women, and varicocele in men, it is called Nutcracker Syndrome (NCS). ${ }^{2}$ It has been suggested that NCS is frequently a disease of tall children with lower body weight. ${ }^{3}$ Patients' age can range from childhood to the seventh decade of life, but most patients become symptomatic in the second and third decades of their lives. ${ }^{1,4}$ It is not a hereditary phenomenon, although coincidental cases in siblings have been described. ${ }^{4}$ The diagnosis of the syndrome is often difficult, and the criteria for diagnosis and treatment are not well established. ${ }^{5}$

The celiac trunk (CeT) and SMA are the two widest vessels arising separately from the ventral aorta. ${ }^{6}$ CeTs exhibited classic trifurcation into the left gastric, common hepatic, and splenic arteries in only $87.7 \%$ of patients. ${ }^{6}$ A celiacomesenteric trunk
Correspondence: Nabil A Al-Zoubi Department of Surgery, Jordan University of Science and Technology, PO Box 3030, Irbid 221 10, Jordan

Tel +962795774637

Email dr_nabeil_z3bi@yahoo.com 

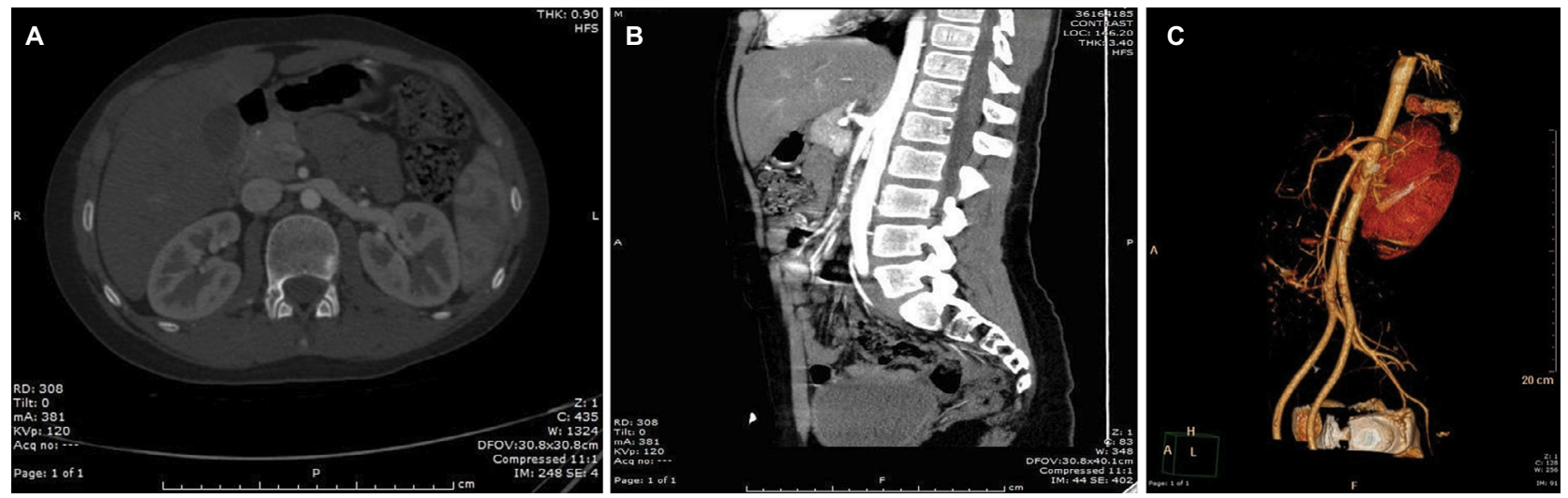

Figure I Axial and sagittal CTA with 3D reconstruction.

Notes: (A) Axial CTA shows significant decrease in diameter of the LRV as it crosses between the aorta and the anomalous CMT. (B) Sagittal CTA shows a very acute aortomesenteric angle, with resultant compression of the LRV by SMA originating from the CMT. (C) Left anterior inferior oblique view of the NCP using 3D reconstruction of the same CTA.

Abbreviations: CMT, celiacomesenteric trunk; CTA, computed tomography-angiography; LRV, left renal vein; NCP, Nutcracker phenomenon; SMA, superior mesenteric artery.

(CMT), with the celiac and SMA having a common origin from the aorta, accounts for less than $1 \%$ of all splanchnic artery anomalies, and it is estimated to have an incidence of $0.25 \%$, with only a few patients with CMT disease having been reported in the literature in a separate series. ${ }^{7-9}$

This article presents a case of a 14-year-old boy who presented with hematuria caused by a rare entity - NCS associated with CMT anomaly. To the best of our knowledge, NCS associated with CMT has not been previously reported.

\section{Case presentation}

A 14-year-old boy with no significant past medical history presented with 3 years of hematuria, which was painless, total, gross, without clots, and continuous every time he voided. He had no burning sensation upon micturition, no frequency, no urgency, and no poor stream. He visited the urology clinic at King Abdullah University Hospital. His vital signs were normal and his physical examinations were unremarkable. His body mass index (BMI) was $19.06 \mathrm{~kg} / \mathrm{m}^{2}$ and serum hemoglobin was $13.1 \mathrm{~g} / \mathrm{dL}$. Kidney function tests including serum electrolytes were normal. Urine analysis showed abundant isomorphic red blood cells, and culture showed no growth. He underwent an ultrasound urinary system that showed no abnormalities. The patient then underwent intravenous pyelogram (IVP) at another hospital and it was normal. He was then scheduled for diagnostic cystourethroscopy which showed normal bladder wall with no gross lesions. Urine cytology was taken, and there was no evidence of high-grade tumors. The patient underwent further evaluation with CT imaging of abdomen and pelvis with IV contrast showing reduced aortomesenteric angle with entrapment of the LRV between the aorta and the SMA with significant stenosis featuring anterior NCS. As part of further anatomical evaluation, a CT-Angiogram (CTA) was performed, which revealed that both SMA and CeT shared the same origin (CMT) (Figure 1). Hemodynamic parameters like duplex ultrasound and renocaval pressure gradient were not available. This case was presented for a discussion with our vascular team, following which conservative management over surgical intervention was chosen.

\section{Consent}

The parents of the patient gave their written informed consent for the case report to be published.

\section{Discussion}

The LRV entrapment between the SMA and aorta was first described by El Sadr and Mina in 1950. ${ }^{4}$ Pournasiri referred to the disorder as NCS in 1972. ${ }^{1}$

Hematuria is a common urinary abnormality with a prevalence of $0.5 \%-2.0 \%$ among school-age children. The prevalence of NCS in children with hematuria is unknown. ${ }^{10}$ It is known that NCS is an uncommon cause of gross or microscopic hematuria from nonglomerular origin. ${ }^{10,11}$ However, hematuria is the most common presentation of this syndrome. NCS presentation depends on the severity of the LRV hypertension which ends to the rupture of the thinwalled septum between the small veins and the collecting system in the renal fornix..$^{1,4}$

The diagnostic criteria of NCS have not been established yet, and there is no simple procedure for a definitive diagnosis at present. ${ }^{7}$ Although it has been suggested that selective left renal venography is the standard procedure for the diagnosis of NCS, we did not use this technique in our patients because of its invasiveness and the burden of radiation that 
it entails. ${ }^{3}$ Several imaging methods are used to diagnose NCS, including Doppler ultrasonography, CTA, and magnetic resonance angiography. ${ }^{12}$ In CTA, the LRV often shows a disparity in caliber between the part distal to the aorta and the part precisely in front of the aorta (beak sign) (Figure 1). Furthermore, a decreased aortomesenteric angle and a shortened aortomesenteric distance can suggest the diagnosis (Figure 1). ${ }^{13}$ It also provides high-quality 3D-reconstructed images and allows noninvasive assessment of normal and abnormal anatomy of $\mathrm{CeT}^{2}$ Since static images will not be able to confirm hemodynamic significance or differentiate between NCP and NCS, the correlation between imaging evidence of LRV compression and clinical symptoms remains a challenge. ${ }^{4,14}$ In this particular case, the inclusion of a CTA in the setting of hematuria was invaluable in locating the source of aberrant vasculature from the CMT.

NCS is a rare condition, which if left untreated may cause thrombosis of the LRV and damage to the left kidney. ${ }^{12,14}$ Although underdiagnosed, there are several treatment options. ${ }^{14}$ The patients diagnosed with NCS should be managed according to their age and clinical symptoms. Usually, patients younger than 18 years of age are more frequently treated conservatively, since a majority of such patients will show complete remission in their clinical symptoms including hematuria because of the development of collateral veins and increase in BMI, which is an important factor in the changing renal hemodynamics. ${ }^{1,2,3,15}$ NCS patients with mild hematuria or with spontaneous resolution of hematuria can be managed with conservative therapy. However, both stenting and open surgical intervention for correcting anatomical anomaly, including procedures such as transposition of the LRV or SMA, nephropexy, intravascular and extravascular stent implantation, gonadocaval bypass, renal autotransplantation, and nephrectomy, are indicated for patients with significant pain, renal insufficiency, and severe, persistent life-threatening hematuria. ${ }^{1,2,4}$ However, selection criteria are not well defined. ${ }^{4}$

The diagnosis of CMT has often been reported during autopsy or accidentally during angiography or abdominal CT scanning. ${ }^{7}$ To date, only 10 patients with CMT disease have been reported in six separate series. ${ }^{8}$ This anomaly has been reported to be associated with clinical conditions like chronic occlusive disease, compression by abdominal aorta aneurysm, celiac compression syndrome, or rarely large gastrointestinal infarction due to thrombosis of the $\mathrm{CMT}^{7}$ This variation is important clinically since awareness of such variation on the part of the surgical team before surgery can help avoid iatrogenic arterial injury, particularly in centers where angiographic studies are not performed regularly.?

In this particular case, we managed the patient conservatively with close follow-up as recommended by the literature, since many studies demonstrated spontaneous resolution of hematuria in children with NCS. ${ }^{3}$ This could be explained by increased BMI, which is an important factor in the changing renal hemodynamics, and due to the development of collateral veins that will reduce the LRV hypertension. ${ }^{1,15}$

Despite there being no previous cases, some theoretical problems should be considered. As this is a rare association between CMT anomaly and NCS, the question of whether conservative management is applied to this variant is not known. Furthermore, if a new therapeutic intervention like the endovascular approach, which involves stenting of the $\mathrm{LRV}$, is decided, taking into account this anatomical external pressure from the CMT on the LRV, then it may not turn out to be feasible (the stent radial force may not overcome the external pressure by CMT and SMA which results in stent fracture). Additionally, awareness of this anatomical constraint may help avoid or reduce the chance of surgical iatrogenic injuries.

\section{Conclusion}

This is an extremely rare case of a 14-year-old boy with gross asymptomatic hematuria caused by anterior NCS in association with CMT anomaly. Whether this abnormal anatomy is the cause of NCS or just an association should be investigated. Moreover, awareness of this anatomical constraint may help in planning therapeutic options and reducing the risk of surgical iatrogenic injuries.

\section{Author contribution}

All authors contributed toward data analysis, drafting and critically revising the paper and agree to be accountable for all aspects of the work.

\section{Disclosure}

The authors report no conflicts of interest in this work.

\section{References}

1. Pournasiri $Z$. The nutcracker syndrome as a rare cause of chronic abdominal pain: a case report. J Compr Ped. 2016;7(3):39741.

2. Sawant DA, Moore TF. An unusual course of segmental renal artery displays a rare case of hilar nutcracker phenomenon. Case Rep Med. 2015;10:1-5.

3. Fitoz S, Ekim M, Ozcakar ZB, Elhan AH, Yalcinkaya F. Nutcracker syndrome in children: the role of upright position examination and superior mesenteric artery angle measurement in the diagnosis. $J$ Ultrasound Med. 2007;26:573-580. 
4. Kurklinsky AK, Rooke TW. Nutcracker phenomenon and nutcracker syndrome. Mayo Clin Proc. 2010;85(6):552-559.

5. Novaes LF, da Silva Saguia LN, Di Migueli CA, et al. Young woman with nutcracker syndrome without main clinic manifestation: Hematuria-Case report. Int J Surg Case Rep. 2017;31:225-228.

6. Kara E, Celebi B, Yildiz A, Ozturk N, Uzmansel ID. An unusual case of a tortuous abdominal aorta with a common celiacomesenteric trunk: demonstrated by angiography. Clinics. 2011;66(1):169-171.

7. Manyama M, Lukanima A, Gesase A. A case of celiacomesenteric trunk in a Tanzanian man. BMC Res Notes. 2013;6:341.

8. Ailawadi G, Cowles RA, Stanley JC, et al. Common celiacomesenteric trunk: Aneurysmal and occlusive disease. J Vasc Surg. 2004;40: 1040-1043.

9. Lee V, Alvarez MD, Bhatt S, Dogra VS. Median arcuate ligament compression of the celiomesenteric trunk. J Clin Imaging Sci. 2011; $1: 8$.
10. Shin JI, Park JM, Lee JS, Kim MJ. Effect of renal Doppler ultrasound on the detection of nutcracker syndrome in children with hematuria. Eur J Pediatr. 2007;166:399-404.

11. Preza Fernandes J, Amorim R, Gomes MJ, Oliveira V, Reis A, RibeiroCastro J. Posterior nutcracker syndrome with left renal vein duplication: a rare cause of haematuria in a 12-year-old boy. Case Rep Urol. 2012;10:1-4.

12. Nickavar A. Nutcracker syndrome; a rare cause of hematuria. J Nephropathol. 2016;5(4):144-145.

13. Inal M, Daphan BU, Bilgili MYK. Superior mesenteric artery syndrome accompanying with nutcracker syndrome: a case report. Iran Red Crescent Med J. 2014;16(10): 14755.

14. Mahmood SK, Oliveira GR, Rosovsky RP. An easily missed diagnosis: flank pain and nutcracker syndrome. BMJ Case Rep. 2013;10:1-3.

15. Tsumura K, Yoshida K, Yamamoto S, Takahashi S, Iida K, Enomoto Y. A case of the nutcracker syndrome developed after delivery. Case Rep Urol. 2014;10:1-5.
The International Journal of Nephrology and Renovascular Disease is an international, peer-reviewed open access journal focusing on the pathophysiology of the kidney and vascular supply. Epidemiology, screening, diagnosis, and treatment interventions are covered as well as basic science, biochemical and immunological studies. The manuscript management system is completely online and includes a very quick and fair peer-review system, which is all easy to use. Visit http://www. dovepress.com/testimonials.php to read real quotes from published authors. 